\title{
Retroperitoneal Liposarcoma: A Case Report and Review of Literature
}

\author{
By Dr. Elize Isabella Wethmar
}

Introduction- Liposarcomas are rare malignant tumours of adipocytic differentiation and are classified under the soft tissue sarcoma subtype histologically. Retroperitoneal liposarcoma (RPL) has an incidence of 1 per 2.5 million individuals, and the retroperitoneum is the second most common site for a liposarcoma, following the lower limb as the most common site. The retroperitoneum is a space that can easily expand;therefore tumours grow in this space without any symptoms until they are very large.

This case study reports a patient diagnosed with a retroperitoneal liposarcoma treated with primary radical surgery and the patient is currently being followed-up in our unit with close monitoring and Computed Tomographic (CT) imaging. The patient consented to the reporting of this case.

GJMR-F Classification: NLMC Code: WJ 768

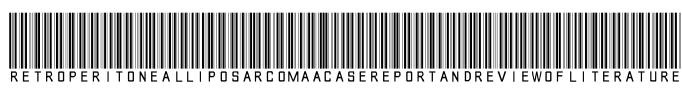

Strictly as per the compliance and regulations of:

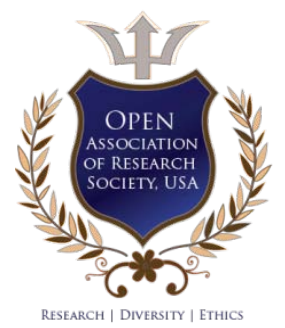

(c) 2021. Dr. Elize Isabella Wethmar. This is a research/review paper, distributed under the terms of the Creative Commons Attribution-Noncommercial 3.0 Unported License http://creativecommons.org/licenses/by-nc/3.0/), permitting all non-commercial use, distribution, and reproduction in any medium, provided the original work is properly cited. 


\title{
Retroperitoneal Liposarcoma: A Case Report and Review of Literature
}

\author{
Dr. Elize Isabella Wethmar
}

\section{INTRODUCTION}

iposarcomas are rare malignant tumours of adipocytic differentiation and are classified under the soft tissue sarcoma subtype histologically. Retroperitoneal liposarcoma (RPL) has an incidence of 1 per 2.5 million individuals, and the retroperitoneum is the second most common site for a liposarcoma, following the lower limb as the most common site. The retroperitoneum is a space that can easily expand;therefore tumours grow in this space without any symptoms until they are very large.

This case study reports a patient diagnosed with a retroperitoneal liposarcoma treated with primary radical surgery and the patient is currently being followed-up in our unit with close monitoring and Computed Tomographic (CT) imaging. The patient consented to the reporting of this case.

\section{il. Case Report}

A 70-year-old female patient was referred to our unit for assessment of anasymptomatic solid left sided adnexal mass seen on CT scan during evaluation for aurological complaint.

At the time of the referral the mass measured $5.5 \times 4.9 \times .7 .2 \mathrm{~cm}$ with a macroscopic fat and a large soft tissue component on CT-scan assessment. Tumour markers were all essentially normal, CA 125 of $15 \mathrm{U} / \mathrm{mL}$, CA 19.9 of $\mathrm{U} / \mathrm{mL}$, CEA of $2.8 \mathrm{ug} / \mathrm{L}$ and the AFP $13.4 \mathrm{k} / \mathrm{U}$, which is a slightly raised level. During this assessment, an excisional procedure was offered to the patient, but due to the Covid 19 pandemic the patient opted to wait with a surgical procedure and follow-up at a later stage.

The patient presented 9 months later for reassessment. During this time the mass had increased significantly in size and had become symptomatic and palpable in the left lower quadrant of her abdomen. On repeat CT-scan the mass appeared to have significantly increased in size, measuring $12 \times 8 \times 14 \mathrm{~cm}$. The mass still had a predominantly solid appearance and was strongly associated to the left ovary and likely ovarian in origin. Ovarian cancer markers were repeated and as above stayed within normal limits. Due to the appearance of the lesion and the very rapid growth, a non-benign lesion, possibly of ovarian origin was suspected and an explorative/staging laparotomy was

Author: Life the Glynnwood Hospital, Benoni, South Africa. e-mail: elize.wethmar@gmail.com offered to the patient. The patient consented to the procedure. A mid-line laparotomy was performed, and a large retroperitoneal mass was found. This mass was adherent to the left ovary, left ureter, left psoas muscle and external iliac vessels as well as the sigmoid colon. With careful anatomical dissection the mass was resected, and a full staging laparotomy and lymph node sampling of the left pelvic and para-aortic lymphnodes was performed. The specimen comprised of a $527 \mathrm{~g}$ encapsulated, lobulated portion of tissue, with a histological diagnosis of a well differentiated liposarcoma, sclerosing variant. No areas of dedifferentiated liposarcoma were noted and the margins were clear of tumour. All resected lymph nodes were negative for metastatic disease. The patient had an uneventful post-operative recovery.

Although the retroperitoneal liposarcoma appears to have been removed completely in this patient, local recurrence is likely. Since there is currently no evidence that radiotherapy or chemotherapy improves survival rates or recurrence rates of the disease in the immediate adjuvant setting, the patient did not receive any adjuvant therapy and is being monitored closely with clinical examinations and 3 monthly CT-scans to evaluate for any local or distant recurrent disease.

\section{ili. Discussion}

\section{a) Surgical and Anatomical features}

The retroperitoneum and the preperitoneum forms the extraperitoneal space, which is the portion of the pelvis and abdomen which does not lie within the peritoneal space. The retroperitoneal space is an almost virtual and expandable space, defined anteriorly by the peritoneal extensions anchoring the transverse colon, the small bowel as well as the ${ }^{1}$ ascending and descending colon, part of the duodenum, part of the pancreas and part of the liver ${ }^{123}$. The retroperitoneum contains the kidneys, the adrenal glands, the pancreas, part of the duodenum, ascending and descending colon, the abdominal aorta and vena cava (dividing into the common iliac, external and internal iliac arteries and veins respectively), the abdominal lymphnodes groups and tracts, six major nerves and the autonomic lumbar nerve chains and the connective tissue of fasciae, with the White line of Toldt as the fusion between the mesocolon and the posterior retroperitoneum. 
Treatment of most conditions involving the retroperitoneum requires surgical intervention. A complete and thorough understanding of the anatomy of the structures involving the retroperitoneum is prudent in avoiding inadvertent damage to underlying structures. Complications may arise from inadvertent damage to structures located within the retroperitoneum during surgical manipulation or instrumentation ${ }^{4}$.

\section{b) Histopathological features}

Retroperitoneal liposarcomas usually consist of a large, well-circumscribed, lobulated mass. Variable consistencies are present, from yellow to firm grey to gelatinous areas, depending on the proportion of fat, fibrous and myxoid components. Larger retroperitoneal tumours appear more heterogeneous, often containing foci of fat necrosis and punctate haemorrhages ${ }^{5}$.

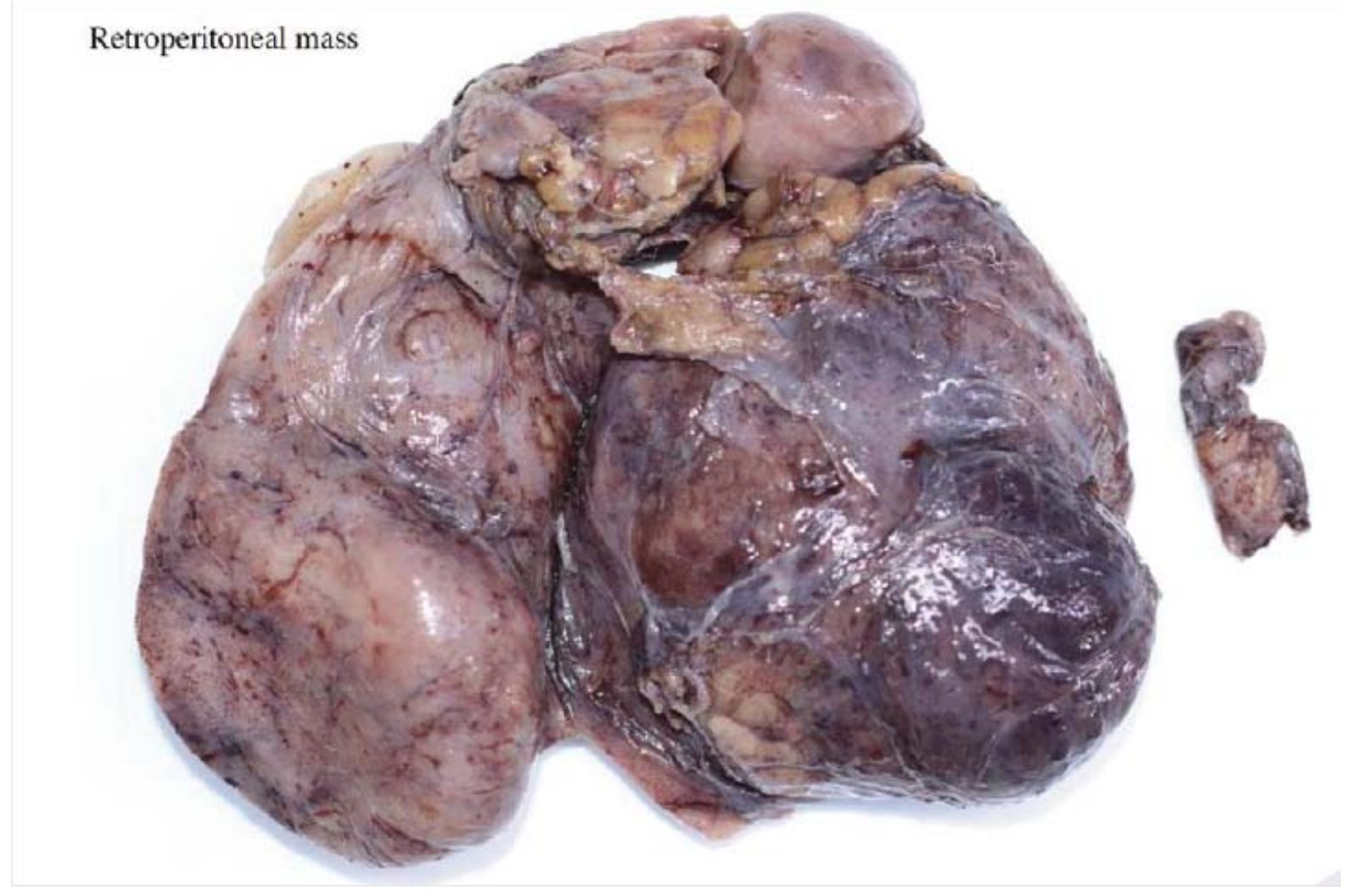

Figure 1: Macroscopic appearance of the excised retroperitoneal liposarcoma

As in many tumours, the histological classification of liposarcoma has evolved over the past several decades, mostly owing to the advances in our understanding of molecular genetics.

The most recent World Health Organisation (WHO) classification system (2020) recognized five different types of major liposarcoma subtypes, differentiated by distinctive morphologies and unique genetic findings 5 :

- Atypical lipomatous tumour (ALT)/ well differentiated liposarcoma (includes adipocytic [or lipoma like], sclerosing and inflammatory variants)

- Dedifferentiated liposarcoma

- Myxoid liposarcoma

- Pleomorphic liposarcoma

- Myxoid pleomorphic liposarcoma

In atypical lipomatous tumour/well differentiated liposarcoma adipocytic variant consists out of cells that vary substantially in size as well as cells that have nuclear atypia in fat or spindle cells. Lipoblasts can be present in various numbers, but the presence or absence is not necessary for the diagnosis of a liposarcoma. Sclerosing atypical lipomatous tumour presents second most frequently and is most often seen in the retroperitoneum or spermatic cord. The most important histological finding is scattered bizarre stromal cells, showing marked nuclear hyperchromasia. Inflammatory ALT represents the rarest subtype, occurring most often in the reproperitoneum ${ }^{5}$. 


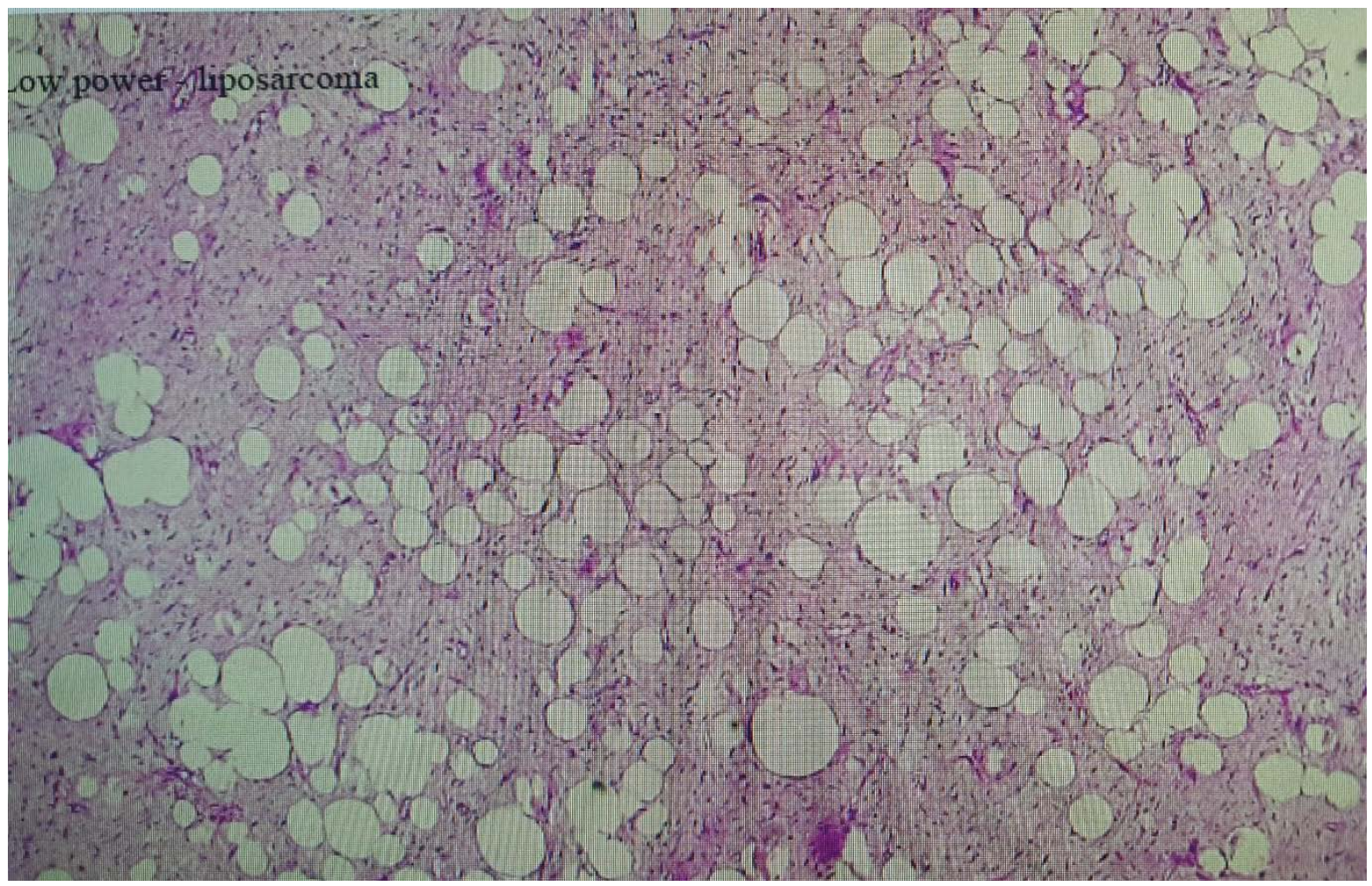

Figure 2: Low power microscopy view demonstrating a deifferentiated liposarcoma

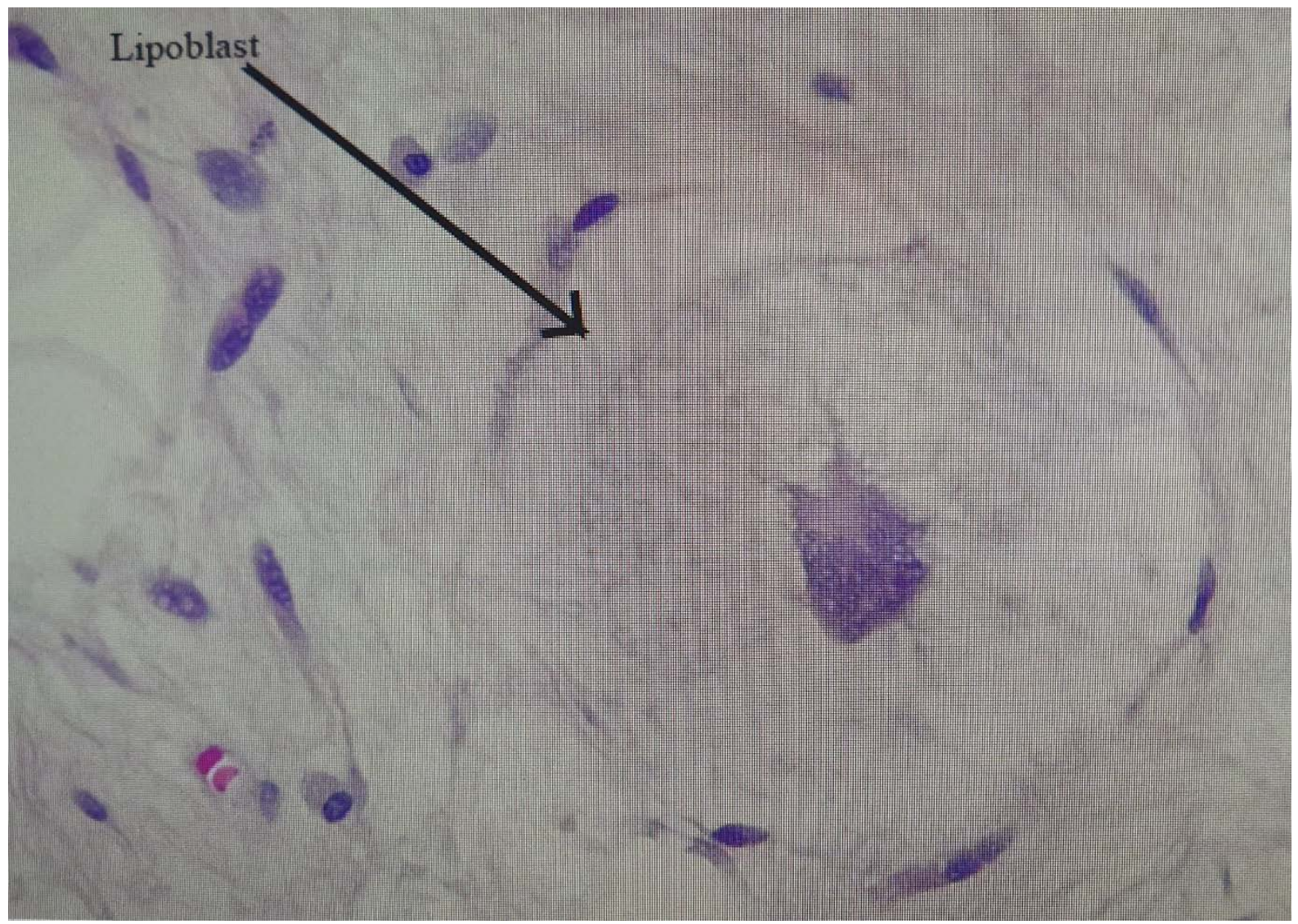

Figure 3: A microscopic view of a lipoblast, which may or may not be present in a liposarcoma 


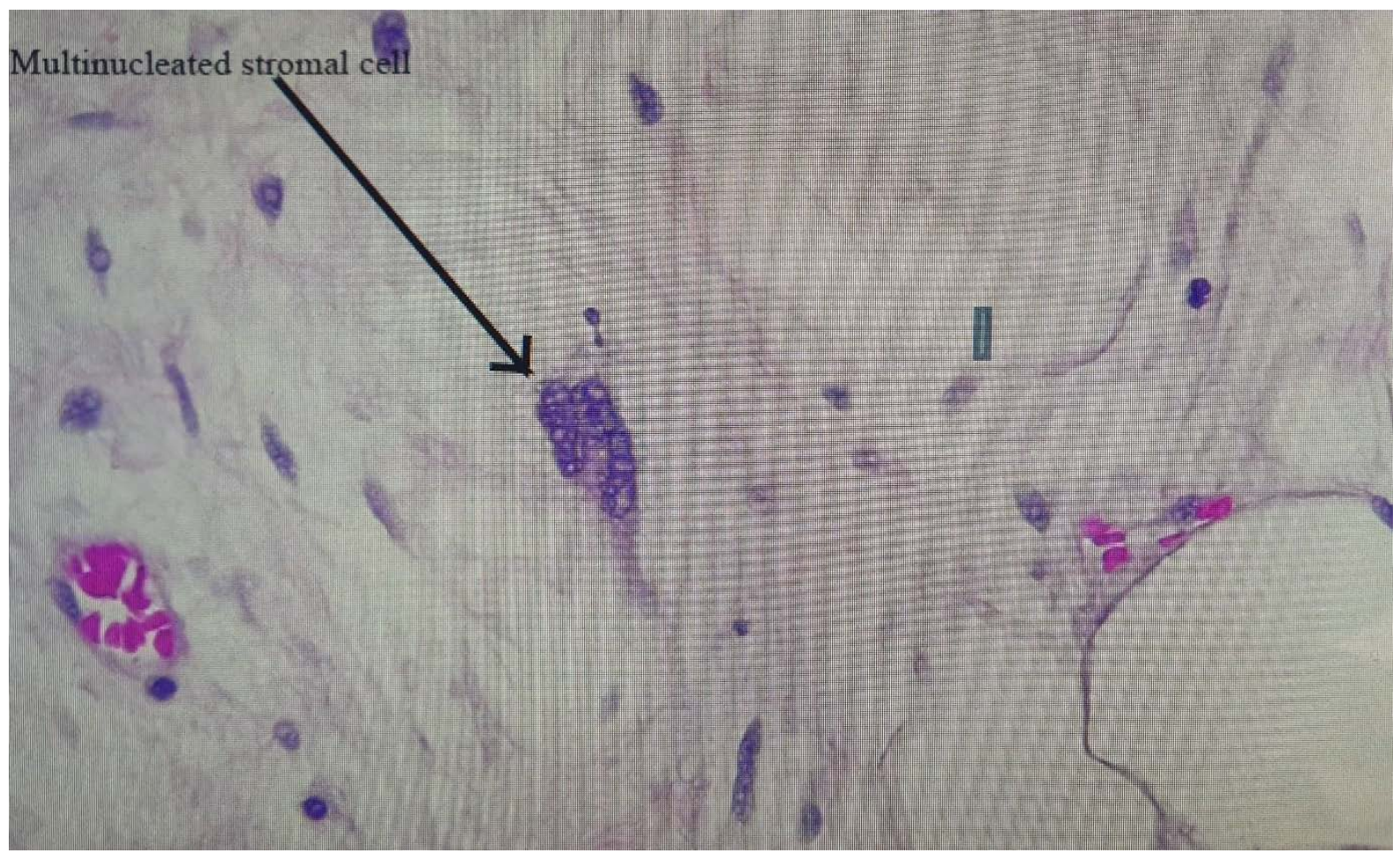

Figure 4: A multinucleated stromal cell which is present in a Sclerosing atypical lipomatous tumour during microscopy

Dedifferentiated liposarcoma can arise as a synchronous lesion in $90 \%$ of cases and as metachronous lesion in $10 \%$ of cases $^{6}$. These tumours exhibit a wide morphological spectrum and histologically show areas of high grade, poorly differentiated sarcoma resembling high-grade myxofibrosarcoma, fibrosarcoma, malignant solitary fibrous tumour or pleomorphic sarcoma not otherwise specified. Dedifferentiated liposarcomas can be of variable histological grade ${ }^{5}$. Dedifferentiated liposarcomas is an aggressive disease, arising most commonly in the retroperitoneum and is associated with high rates of local and metastatic recurrence and disease specific mortality ${ }^{7}$.

Use of the term atypical lipomatous tumour is determined by tumour location and resectability. In locations such as the retroperitoneum, it is usually impossible to obtain a wide tumour free surgical margin of more than $2 \mathrm{~cm}$, thus local recurrence is common and leads to mortality, seen in the absence of dedifferentiation or metastases ${ }^{8}$.

\section{c) Radiological features}

The introduction of computed tomography (CT), magnetic resonance imaging (MRI), and ultrasonography have greatly enhanced our capability to make the diagnosis of abdominopelvic neoplasms and determine and direct treatment, as well as observe the progress of the neoplasm and effect of treatment over time. Identification of a retroperitoneal mass at imaging is a challenging task for radiologists, however the presence of fat within a retroperitoneal lesion is helpful in refining the differential diagnosis ${ }^{9}$. It is easy to recognize fat within a lesion due to its characteristic imaging appearance:

- On ultrasound appearance it is hyperechoic and may demonstrate posterior acoustic shadowing

- Computed Tomographic (CT) imaging derives contrast parameters predominantly from the physical properties of tissue - in combination with high resolution spiral $\mathrm{CT}$, this provides accurate attenuation measurement, with -10 to -100 HU9corresponding to fat $^{10}$

- Magnetic resonance imaging (MRI) has lower spatial resolution than CT imaging, but better soft tissue contrast and greater sensitivity in detection of microscopic fat. The two MRI techniques for the identification of fat within a structure are fat saturation and chemical shift imaging

It can be difficult to localize large abdominal masses to an anatomical space and to accurately identify the organ of origin thus to determine whether the lesion arises from a retroperitoneal organ, or from the soft tissue ${ }^{10}$. If the mass is surrounded by the parenchyma of the organ, it undoubtedly arises from the organ, however if the mass originates at the margin of the organ, it is more difficult the determine the origin. The interface between the mass with the adjacent organ can provide insight as to whether it displaces the organ or arises from the organ (Table 1). 
Table 1: Positive signs indicating that a Retroperitoneal Tumour Arises from an Adjacent Organ ${ }^{9}$

\begin{tabular}{|l|l|}
\hline \multicolumn{1}{|c|}{ Sign } & \multicolumn{1}{c|}{ Definition } \\
\hline Beak sign & $\begin{array}{l}\text { Sharp beak shape of organ of origin occurs at the edge of the interface with } \\
\text { the tumour }\end{array}$ \\
\hline Embedded organ Sign & Organ of origin is encased by the tumour, without a sclerotic interface \\
\hline Phantom (invisible) organ sign & Organ of origin is obliterated by the tumour \\
\hline Prominent feeding artery sign & Large feeding arteries of a hypervascular tumour point to the organ of origin \\
\hline
\end{tabular}

A CT image of a lipoma will reveal a welldefined homogenous mass with fat attenuation. Areas of soft-tissue attenuation may be seen within the tumour and may represent fat necrosis, septa or normal adjacent structures. If a predominantly solid soft-tissue component or adjacent organ invasion is present, a liposarcoma should be suspected. The imaging characteristics of liposarcomas differs, depending on the histological subtype. Well-differentiated liposarcomas appear as well defined predominantly fat- containing lesions with minimal soft tissue attenuation and commonly contain septa - the appearance may be indistinguishable from a lipoma ${ }^{9}$ and therefore aretroperitoneal purely fatty lesion should be considered a liposarcoma rather than a lipoma until proven otherwise with histological confirmation ${ }^{1011}$. Dedifferentiated liposarcomas appear remarkably similar to well-differentiated liposarcomas of CT imaging, and dedifferentiation is suggested by focal nodular non-lipomatous regions larger than $1 \mathrm{~cm}^{12}$.

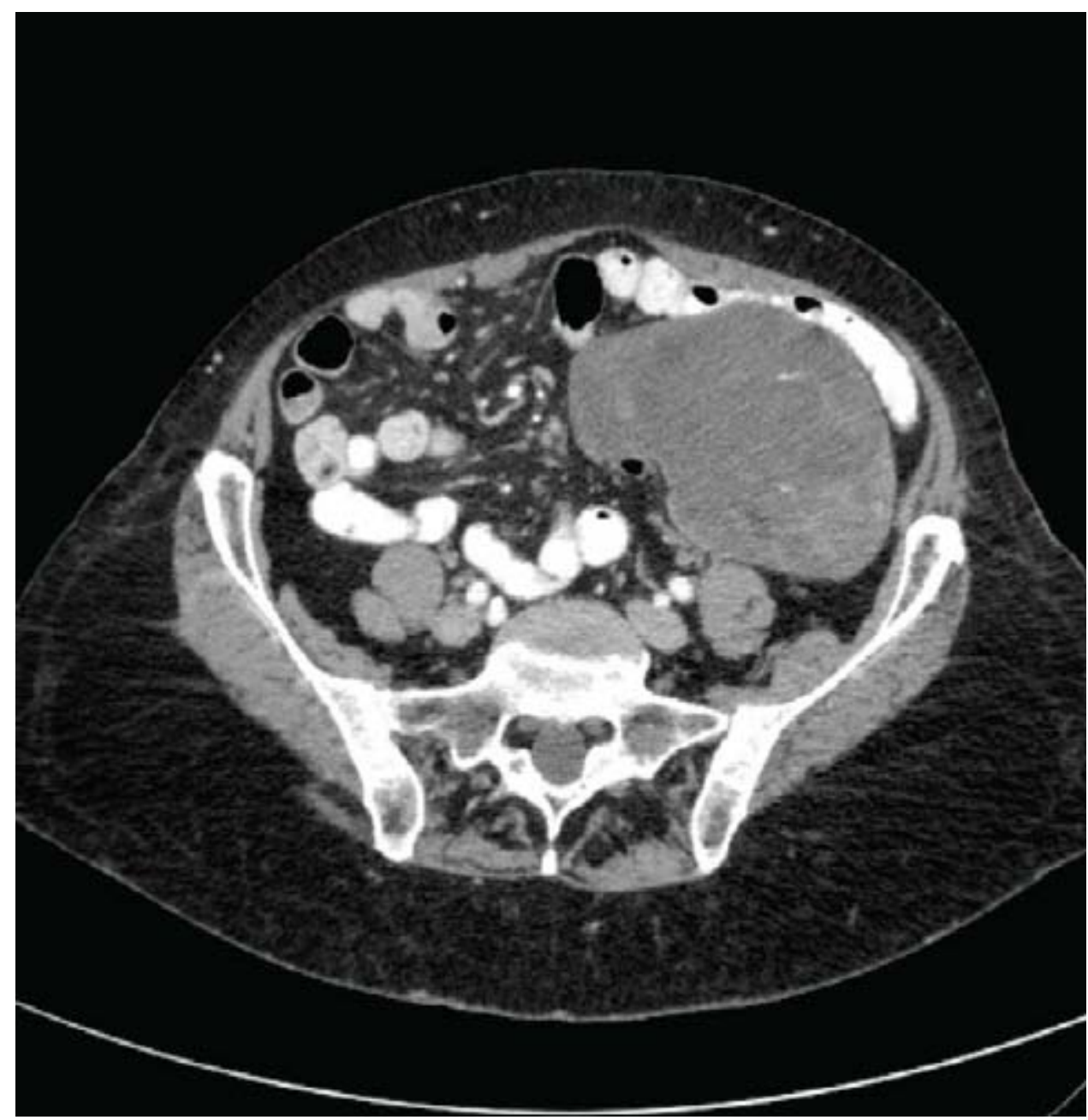

Figure 5: Transverse CT-scan image demonstrating a liposarcoma 


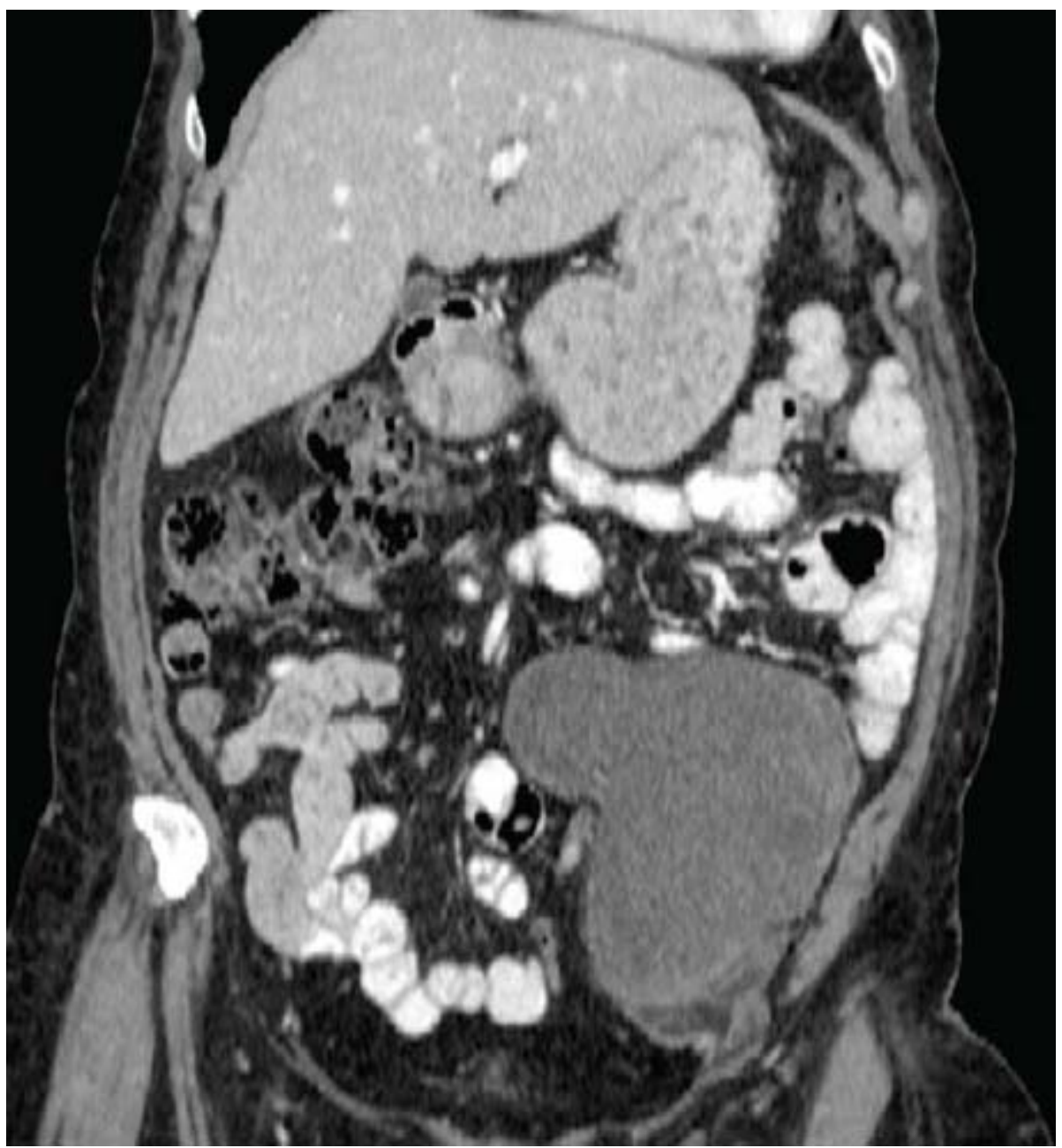

Figure 6: Coronal CT scan image demonstrating the liposarcoma

\section{d) Treatment options}

\section{i. Surgery}

Surgery is the mainstay of treatment for nonmetastatic retroperitoneal sarcoma ${ }^{8}$. If possible, macroscopically complete resection of tumour should be aimed for and this can lead to radical surgery requiring en-bloc removal of adjacent structures. If the pre-treatment diagnosis can be made with certainty, based on radiologic and clinical findings and complete resection is deemed possible, pre-treatment biopsy is not advised and has no value ${ }^{13}$. If radiologic investigations suggest a pathology that does not require primary surgery (e.g. lymphoma, Ewing Sarcoma, GIST) or the incomplete resection is expected, biopsy will be necessary to plan alternative treatment. Image guided core biopsy is advised and preferred over open or laparoscopic approaches, which may be associated with tumour spillage and compromise future surgical strategy by altering tissue planes ${ }^{14}$.

The removal of the entire tumour with a margin of normal tissue is usually not possible in large retroperitoneal liposarcomas due to the presence of adjacent large vessels, nerves and bony structures, leading to local recurrence in the abdomen, which constitutes the cause of death in three out of four patients ${ }^{15}$. High grade, dedifferentiated tumours are at a higher risk to recur and spread systemically, so even if extensive surgery with adequate margins is achieved, the prognosis remains dismal, querying the fact whether a patient should be exposed to the morbidity of extensive surgery if the mortality in dedifferentiated liposarcoma remains high irrespective of treatment.

\section{ii. Radiation Therapy}

Currently there is no convincing evidence for the role of radiotherapy (RT) in the adjuvant setting for the management of RLS. Several authors have analysed the data from the surveillance, epidemiology, and end result (SEER) data base in order to define the role of adjuvant $R T$ in RLS and as a general finding it did not improve survival or did so in a subgroup of patients with stage I disease only. To date, no randomised trials have been completed or published comparing surgery alone with combined surgery and RT. Pre-operative RT in certain settings is showing some promise, however further studies and data would be needed. 


\section{iii. Systemic therapy}

Chemotherapy has an established role in the palliative management of advanced or metastatic soft tissue sarcoma ${ }^{8}$. Active agents include the anthracyclines (doxorubicin and epirubicin) and the alkylating agent ifosfomide ${ }^{16}$. In patients with resistant disease, gemcitabine, docetaxel, trabectedin and pazopanib were established as effective second- or third-line options in the recent years ${ }^{17}$.

The response of liposarcoma to chemotherapy differs according to histological subtype and grade $^{8}$. Well-differentiated and dedifferentiated liposarcoma respond poorly to systemic therapy, therefore novel molecular targets will have to be identified to explorenew possibilities for treatment. MDM2 and CDK4 targeted therapy as well as the tyrosine kinase inhibitor Sunitinib is currently showing promise in treatment of $R L S^{8}$.

\section{Conclusion}

Retroperitoneal liposarcoma is a rare malignancy and challenging to diagnose, treat and monitor for recurrence. The presence of ahigh-grade dedifferentiated component does make the disease more aggressive, but even well differentiated tumours can be difficult to manage and recurrences can be widespread and higher grade than the primary tumour.

Even though the mainstay of treatment is surgery, multi-disciplinary discussion is paramount, especially in view of anatomically irresectable sights and potential future benefits of non-surgical therapies. As with any rare malignancy national and international collaboration is encouraged to learn from each other and improve management of retroperitoneal liposarcoma.

\section{References Références Referencias}

1. Mirilas, P. and Skandalakis, J., 2010. Surgical Anatomy of the Retroperitoneal Spaces Part II: The Architecture of the Retroperitoneal Space. The American Surgeon, 76(1), pp.33-42.

2. Mirilas, P. and Skandalakis, J., 2009. Surgical Anatomy of the Retroperitoneal Spaces-Part I: Embryogenesis and Anatomy. The American Surgeon, 75(11), pp.1091-1097.

3. Liles, J., Tzeng, C., Short, J., Kulesza, P. and Heslin, M., 2009. Retroperitoneal and Intra-Abdominal Sarcoma. Current Problems in Surgery, 46(6), pp.445-503.

4. Mirilas, P. and Skandalakis, J., 2010. Surgical Anatomy of the Retroperitoneal Spaces, Part IV: Retroperitoneal Nerves. The American Surgeon, 76(3), pp.253-262.

5. Anderson, W. and Doyle, L., 2021. Updates from the 2020 World Health Organization Classification of Soft Tissue and Bone Tumours. Histopathology.

6. Coindre, J., Pédeutour, F. and Aurias, A., 2009. Welldifferentiated and dedifferentiated liposarcomas. Virchows Archiv, 456(2), pp.167-179.
7. Lee, A., Thway, K., Huang, P. and Jones, R., 2018. Clinical and Molecular Spectrum of Liposarcoma. Journal of Clinical Oncology, 36(2), pp.151-159.

8. Matthyssens, L., Creytens, D. and Ceelen, W., 2015. Retroperitoneal Liposarcoma: Current Insights in Diagnosis and Treatment. Frontiers in Surgery,

9. Shaaban, A., Rezvani, M., Tubay, M., Elsayes, K., Woodward, P. and Menias, C., 2016. Fat-containing Retroperitoneal Lesions: Imaging Characteristics, Localization, and Differential Diagnosis. RadioGraphics, 36(3), pp.710-734.

10. Pubs.rsna.org. 2021. Fat-containing Retroperitoneal Lesions: Imaging Characteristics, Localization, and Differential Diagnosis | RadioGraphics. [online] Available at: <https://pubs.rsna.org/doi/10.1148/ rg.2016150149> [Accessed 14 April 2021].

11. Song, T., Shen, J., Liang, B., Mai, W., Li, Y. and Guo, H., 2007. Retroperitoneal liposarcoma: MR characteristics and pathological correlative analysis. Abdominal Imaging, 32(5), pp.668-674.

12. Murphey, M., Arcara, L. and Fanburg-Smith, J., 2005. Imaging of Musculoskeletal Liposarcoma with RadiologicPathologic Correlation. Radio Graphics, 25(5), pp. 1371-1395

13. Chew, C., Reid, R. and O'Dwyer, P., 2006. Value of biopsy in the assessment of a retroperitoneal mass. The Surgeon, 4(2), pp.79-81.

14. Hwang, S., Warrier, S., Thompson, S., Davidson, T., Yang, J. and Crowe, P., 2013. Safety and accuracy of core biopsy in retroperitoneal sarcomas. Asia-Pacific Journal of Clinical Oncology, 12(1), pp.e174-e178.

15. Anaya, D., Lahat, G., Wang, X., Xiao, L., Tuvin, D., Pisters, P., Lev, D. and Pollock, R., 2008. Establishing Prognosis in Retroperitoneal Sarcoma: A New Histology-Based Paradigm. Annals of Surgical Oncology, 16(3), pp. 667-675.

16. Krikelis, D. and Judson, I., 2010. Role of chemotherapy in the management of soft tissue sarcomas. Expert Review of Anticancer Therapy, 10(2), pp.249-260.

17. Constantinidou, A., Pollack, S., Loggers, E., Rodler, E. and Jones, R., 2013. The evolution of systemic therapy in sarcoma. Expert Review of Anticancer Therapy, 13(2), pp.211-223. 